\title{
OPINION \\ Decision-making through the lens of a pediatric cancer
}

case

Tamara Danielle Selman, BSc

Class of 2022, Faculty of Medicine, Dalhousie University, Halifax, Nova Scotia, Canada.

\begin{abstract}
It is crucial to obtain a competent individual's informed consent in any medical process, including cancer treatments. However, when it comes to incompetent children, it seems to be favourable, but not necessary, to obtain their assent in medical practice.' This paper considers Christine Harrison's example of Samantha, an eleven-year-old girl that was treated for osteosarcoma in her left arm. Samantha had previously been treated by amputation and a course of chemotherapy. This cancer later metastasized to her lungs, decreasing her chances of remission with aggressive treatment to $20 \%$. Although she wanted to refuse treatment, she was deemed incompetent to make decisions about her cancer care, and her parents adamantly wanted her to continue treatment. ${ }^{7}$ This paper considers physicians' moral obligations in pediatric cancer cases such as Samantha's. I will define assent, the principles of autonomy, beneficence, and competence as it pertains to children. I consider arguments of two opposing views-a child's rights view that argues in favour of Samantha's decision, and a paternalistic view that opposes her. After reviewing the bioethical literature on the risks and benefits of children's decision making in health care, I argue that Samantha's wishes to stop treatment ought to be respected. Throughout the paper, I will use the bioethical principles of respect for autonomy and beneficence to defend my position. Finally, I address potential objections my position may face and conclude.
\end{abstract}

Tt is crucial to obtain a competent individual's Linformed consent in any medical process, including cancer treatment. However, when it comes to incompetent children it seems to be only favourable - not necessary - to obtain their assent in medical practice. ${ }^{1}$ I will consider Christine Harrison's example of Samantha, an eleven-year-old girl that was treated for osteosarcoma in her left arm. ${ }^{7}$ Samantha had previously been treated by amputation and a course of chemotherapy. Although she was cancer free for 18 months, she was self-conscious about her prosthetic and had to give up her cat for fear of infection. This cancer later metastasized to her lungs, decreasing her chances of remission with aggressive treatment to $20 \%$. Although she wanted to refuse treatment, she was deemed incompetent to make decisions about her cancer care, and her parents adamantly wanted her to continue treatment. ${ }^{7}$ Although Samantha's parents legally have the final say in her cancer care, what are physicians' moral obligations in pediatric cancer cases such as Samantha's? After reviewing the literature on the risks and benefits of children's decision-making in health care, I will argue that Samantha's wishes to stop treatment should be respected. This paper will be composed of three sections. In the first, I define the moral status of children, basic bioethical terms, as well as two opposing views that pertain to decisionmaking in pediatric cases. In the second, I apply these definitions to the bioethical principles of respect for autonomy and beneficence to defend my position. In the third, I consider objections that my position faces and conclude.

\section{Section I: Definitions and Assumptions}

First, this paper will assume that the moral status of children is as described by Brennan and Noggle for whom children are of equal moral status as their parents and are not owned by the parent. That is not to say that they have the same rights as their parents, as having certain rights entails that one has a certain role. For example, children cannot drive because that right comes with a responsibility that children cannot fulfill due to their incompetence. ${ }^{3}$ Parents, on the other hand, are stewards. They have a responsibility to exercise their children's rights and refrain from violating these rights, as well as to promote the best interests of the child. ${ }^{3}$ However, parents are often put in situations where they must balance the interests of their other children and the interests of the entire family. I will touch on family interests in the following section and argue that promoting Samantha's best interests does entail respecting her wishes to stop aggressive treatment.

Next, there are four bioethical principles to which health practitioners adhere when administering care: respect for autonomy, beneficence, non-maleficence, and justice. ${ }^{7}$ When addressing issues in pediatric cases, usually the principles of autonomy and beneficence are explored by health practitioners. ${ }^{79}$ The principle of respect for autonomy states that competent individuals have the right to make decisions for themselves, even if these decisions are not in their health-related interests; the principle of beneficence urges health practitioners to act in the best interest of their patients. ${ }^{7}$ I will use these concepts throughout this paper to demonstrate that Samantha's wishes ought to be respected. 
In order to adhere to the principle of respect for autonomy, informed consent is sought by health practitioners in clinical settings. Informed consent allows health practitioners to perform procedures that otherwise would not be acceptable, and it has five components: competence, disclosure, understanding, voluntariness, and token consent. ${ }^{8}$ If any of these five components are not met, the consent is not valid. For the purposes of this discussion, competence is the most relevant component of informed consent. Competence in particular is task and time-dependent and may be met if one can give recognizable reasons for one's decisions. ${ }^{7}$ There are different stages of competence when it comes to minors. Infants and very young children are assumed to lack competence. Then, minors enter into the stage in which they develop competence. Competence, in this stage, is a continuum that needs to be assessed individually, based both on the nature of the procedure and the current stage of cognitive development of the patient. Being in this stage, Samantha may be able to participate to an extent in decisions regarding her care, but not fully. Finally, once minors reach the age of majority (typically around eighteen) they are assumed to have developed full competence. ${ }^{7}$ In pediatric settings, children who are incompetent or are developing competence can give their assent to a procedure. ${ }^{9}$ Assent retains the disclosure and voluntariness requirements of consent, but dispenses with the requirements of competence and understanding which would be expected from an adult. ${ }^{8}$ Although the bioethical literature recommends that a child assent to a procedure, a child's decision to dissent to life-saving treatment, such as that of Samantha, can be overruled. ${ }^{9}$

To conclude the first section, I will outline two opposing views on decision-making in pediatric cases. The first is a paternalistic view, according to which parents ought to make health-related decisions for their children. ${ }^{9}$ If we were to apply the paternalistic view to Samantha's case, Samantha's parents ought to make the final decision on her care, regardless of her dissent. The second view I will call a "children's rights" view and is popular within the bioethical literature. ${ }^{3}$ The children's rights view is an umbrella term for a number of different perspectives that seek to increase the role that children have in their own treatments. Thus, one should recognize that there are many ways in which children's rights views can be interpreted. Some children's rights views may border on the paternalistic view, while others assign the same rights to children as adults. However, children's rights differ from adult rights in the ways outlined by Brennan and Noggle having a right is dependent on having a role that allows you to exercise said right. ${ }^{3}$ Thus, for the purposes of this paper, the children's rights view is nuanced, and argues for stronger respect for a child's consent, but not complete adult rights. Furthermore, in clinical practice, children can dissent to their medical treatments and are part of the decision-making process for their care. However, their dissent may be overruled by their parents or medical staff in life-threatening cases. ${ }^{9}$ When applying the children's rights view to Samantha's case, one must analyze various factors before choosing to accept her dissent. Both the paternalistic and children's rights views appeal to arguments that touch on increasing children's autonomy and the best interest standard, which is the western standard of care when it comes to pediatric cases. ${ }^{5}$

\section{Section II: Analysis}

The following paragraphs will pertain to the principle of respect for autonomy. In pediatric oncology cases such as Samantha's, it is not sufficient to simply state that accepting her decision allows for the principle of respect for autonomy to be achieved. Various arguments pertaining to decision-making in pediatric cases appeal to the principle of respect for autonomy as a reason (but not the sole reason) children should (or should not) be free to make a medically-related decision.

First, as Samantha is a minor, she is not deemed legally competent to make a life-or-death medical decision related to her osteosarcoma. ${ }^{7}$ Her parents are thus required to practice Samantha's autonomy for her. ${ }^{3}$ Supporters of the paternalist view may argue that there is no objective test to assess competence for every single individual, thus we may not truly know that Samantha is competent to make decisions in a pediatric oncology context. ${ }^{9}$ However, a 2006 study by Alderson et al. demonstrated that children with diabetes were able to understand and reach an acceptable level of competence when it came to their illness, and were able to make their informed medical decisions in this context. ${ }^{1}$ Furthermore, as described in section I, competence can be assessed using the recognizable reasons standards. Although this standard is not completely objective, Samantha has given recognizable reasons to discontinue care-- the treatment is aggressive, the prognosis is poor, and she wants to die a peaceful death. Given that competence is context dependent and Samantha has undergone cancer treatments in the past, she may understand how the aggressive cancer treatments make her feel something her parents are unable to do. As explained in section I, this allows Samantha to be awarded certain rights to make this decision, as she has taken on the 
role of a cancer patient, but does not allow her to make any decision she pleases. ${ }^{3}$ This role allows Samantha to understand what it is like to undergo treatment, how this treatment affects her body, her mental state as well as her social context. Therefore, it is reasonable to believe that Samantha has capacity in this case to make a decision regarding her care.

Those that agree with the paternalistic view may argue that Samantha's parents should have the final say in her care because it is important that Samantha's long-term autonomy is respected, not simply her present-day feelings. ${ }^{9}$ Samantha's decision does little to respect her long-term autonomy, as her decision will ultimately lead to her death. But we can also assess one's long-term autonomy by looking to retroactive consent. $^{3}$ An example of retroactive consent as it pertains to cancer care can be given by examining the case of Brian Fies's mother in his book "Mom's Cancer". After looking back at her aggressive treatment to cure her brain cancer, Brian's mother stated that she would not have consented had she known her chances of survival were low and the toll the radiation would take on her body. ${ }^{6}$ Assuming Samantha survives to adulthood, would she give her retroactive consent to the procedure? It is plausible for an adult Samantha to question her parents' decision to continue with her cancer care given the inconvenience of aggressive treatment and the high chance that she will die an arguably unpleasant death. Given the aggressive nature of treatment in cancer cases, it is understandable for adults to refuse life-saving treatment, and instead, choose to live out the rest of their days on their terms. Thus, it may be that in Samantha's case her decision to discontinue treatment does not harm her long-term autonomy.

The following paragraphs will pertain to the principle of beneficence. The best interest standard stems from the principle of beneficence and is the standard western society uses to medically treat children. ${ }^{7}$ This standard is often consequentialist, and usually opts for life-saving treatments as saving one's life is arguably in their best interest. ${ }^{4}$ In some cases, if parents do not consent to life-saving treatments for their children, physicians have the right to intervene in the name of the child's best interest. ${ }^{4}$ There are several ethical issues surrounding the best interest standard. The first is that often children are deemed competent if they make the "right" choice and incompetent if they choose to stop treatment, like Samantha. ${ }^{1}$ Furthermore, this standard can be ambiguous, as it is very difficult to determine what is in one's objective best interests in these tough medical contexts, and it is often left to physicians to make this decision. ${ }^{2}$ Samantha pursuing aggressive cancer treatment may not necessarily be in her overall best interests. There are several reasons that support this statement.

First, De Vries et al. conducted a study in a pediatric oncology setting about what parents and physicians assume to be the best interest of children in this context. During the early stages of diagnosis and treatment, parents deemed survival to be in the child's best interest and were willing to do whatever physicians recommended. However, after the initial shock of the cancer diagnoses subsided, parents and children felt that what is in one's best interests are values outside of medical context like leading a good life, having a sense of control, and maintaining their identities - either through religion or other ways. ${ }^{5}$

The paternalist view on decision-making in healthcare might argue that we trust parents to make decisions that are in their child's best interests in general - for example, which schools they attend, and what food they eat. ${ }^{4}$ Therefore, Samantha's parents are probably the best equipped to know what is in her health interests as well. However, the child's rights view may reply that not only are parents making decisions that benefit Samantha but a decision that benefits their family as a whole, and this may override Samantha's personal interests. ${ }^{4}$ In some cases, it is important to take into account general familial interests when dealing with patients, and it is obvious that Samantha's passing is going to change her family's dynamic and will be extremely difficult on her parents and any siblings she may have. However, Samantha's chances of remission are very low, and it is possible she is going to die with or without treatment. Thus, it may not be in this family's best interests to spend their last few moments with Samantha in a setting where their daughter is clearly uncomfortable and fighting with them.

Finally, Samantha expressed feelings of self-consciousness about her prosthetic, as well as being upset about giving up her cat "Snowy". She had shown extreme discomfort throughout her treatment and even struggled violently, which made the medical staff reluctant to keep administering treatment. Samantha stated that so much had been taken away from her and she was upset that her parents wanted her to give up even more. ${ }^{7}$ Samantha's case can be mirrored through a case presented by Kunin. A.P. was a 12-year-old with an osteosarcoma in his arm, and eventually, after several aggressive treatments that did not seem to prove effective, he expressed the desire to spend more time 
with his family and friends and leave the hospital. ${ }^{8}$ It is important to note that if Samantha were to pursue aggressive treatment, there would be a twenty percent chance of remission. In A.P's case, however, there was no chance of remission thus exposing him to more pain, and toxicity from aggressive treatments might border on child abuse. ${ }^{8}$ Although there is this difference between the two cases, Samantha's chances of remission are arguably low. Perhaps switching to a palliative care setting to die an arguably better death than she would have at the hospital is best.

\section{Section III: Objections and Conclusion}

The following paragraphs will address potential objections one could raise against my position, and offers replies that further justify Samantha's decision to end life-saving treatment.

One may argue that there is a morally significant difference between allowing an elderly person to make a life-ending medical decision and allowing Samantha-although potentially competent, to do the same. This is due to the number of life years lost. For example, if an elderly person such as Brian Fies's mother decides to end an aggressive, possibly futile treatment, what is lost is a few years of life. However, in Samantha's case, although the chances of success in her treatment are low, if she does survive she will gain an entire lifetime.

In reply, while it is true that should Samantha's treatment work she will gain more life years than an elderly person in her position, this argument is not consistent with current medical practice. Ethically, clinicians allow competent young adults and teenagers to make life-ending medical decisions in order to respect their autonomy and non-health related values. There are many life years lost in these contexts as well, but these decisions are justified not based on life years lost or gained.

One may object that if Samantha is able to make a life or death decision regarding her health, then she should also be able to make other decisions about her life that traditionally adults make. ${ }^{9}$ For example, Samantha could be a student at an elementary school, and as most students in elementary school, she would rather be on summer vacation or sleeping in than in the classroom. Given the nature of the arguments in section II, since Samantha goes to school every day, she knows better than her parents about her school environment. Thus, if one day Samantha decides to drop out of elementary school, she should be able to do so. Through this comparison, if one accepts that Samantha can make life or death decisions, one must also accept that Samantha should be able to drop out of school.

In reply, there is a morally significant difference between Samantha's decisions regarding her cancer treatment and her decisions regarding school. The first is, as I have demonstrated above, discontinuing aggressive cancer treatment and allowing Samantha to live out the rest of her days with her family and friends may be in her best interests and might not harm her future autonomy. Dropping out of school, however, is arguably not in her best interests, and can potentially harm her overall autonomy because her opportunities to make choices will be reduced if she does not get an education. To highlight this point further using the retroactive consent argument described in section II, most adults would not give their retroactive consent to drop out of elementary school. Furthermore, competence is context dependent as described above. Although Samantha can make a life or death decision regarding her case, that does not mean that Samantha can make a decision surrounding her education. There are two reasons for this. The first is that Samantha has given recognizable reasons for her wanting to discontinue her care as argued in section II. The second is that there are little to no recognizable reasons for wanting to drop out of elementary school that outweigh the objective benefits of an education. Thus, Samantha can make a life or death decision regarding her cancer care but still is unable to make the decision to drop out of elementary school.

In conclusion, Samantha's wishes to stop treatment for her osteosarcoma should be respected. After considering arguments from both the paternalistic and child's rights views pertaining to autonomy and best interest, I have concluded that physicians have a moral obligation towards Samantha and her decision. Although Samantha is a minor, her autonomy should be respected as she may be competent to make this decision given her context, and her future autonomy might not be harmed in doing so. The best interest standard commonly used in pediatric medicine has flaws that are disadvantageous to Samantha. Samantha has other values aside from her health-related values that contribute to her best interests, and these should be respected by the health practitioners. Samantha's parents eventually decided to respect her wishes to die after consulting with the team of health practitioners as well as an ethics board. They took Samantha home where she was given a new cat and died peacefully. ${ }^{7}$ 


\section{Acknowledgements}

I would like to acknowledge Dr. Lynette Reid from Dalhousie University's Department of Bioethics, Dr. Kirsten Borgerson from Dalhousie University's Department of Philosophy, and Cameron Yetman for their contributions to this paper.

\section{References}

1. Alderson, P., Sutcliffe, K., \& Curtis, K. Children's competence to consent to medical treatment. Hastings Cent Rep 2006;36(6):2534.

2. Birchley, G. Deciding Together? Best Interests and Shared Decision-Making in Paediatric Intensive Care. Health Care Analysis 2013;22(3):203-222.

3. Brennan, S., \& Noggle, R. The moral status of children:
Children's rights, parents' rights, and family justice. Social Theory and Practice 1997;23(1):1-26.

4. Cherry, M. J. Parental authority and pediatric bioethical decision making. J Med Philos, 2010;35(5):553-572.

5. De Vries, M.,C., Bresters, D., \& Kaspers, G. J. L. What constitutes the best interest of a child? views of parents, children, and physicians in a pediatric oncology setting. American Journal of Bioethics: Primary Research 2013;4(2):1-10.

6. Fies, B. (2004). Mom's Cancer by Brian Fies. Retrieved March 10, 2018, from http://www.gocomics.com/moms-cancer

7. Harrison, C., Kenny, N.P., Sidarous, M., \& Rowell, M. Involving children in medical decisions. CMAJ 1997;156:825-8

8. Kunin, H. Ethical issues in pediatric life-threatening illness: Dilemmas of consent, assent, and communication. Ethics and Behavior 1997;7(1):43-57.

9. Ross, L. F. Health care decision-making by children. Is it in their best interest. Hastings Cent Rep 1997;27(6):41-45. 